\title{
Ethische Fallbesprechungen und «Beratung» - kritische Einführung
}

\section{Rouven Porz}

PD Dr. phil., dipl. biol., Leiter der Fachstelle für klinische Ethik des Inselspitals / Spital Netz Bern AG, Mitglied Redaktion Ethik der SÄZ

\begin{abstract}
Ethische Unterstützung durch Fallbesprechung kommt in der Regel dann zum Einsatz, wenn es um moralisch-schwierige Handlungen und Entscheidungen geht. Doch schon die Beurteilung, was «moralisch-schwierig» ist, kann individuell sehr differieren. Und die Organisation und Durchführung ethischer Fallbesprechungen birgt eine Vielzahl von Problemen. Nicht immer sind sie das probate Mittel zur Problemlösung. Über Alternativen sollte nachgedacht werden.
\end{abstract}

\section{Eine neue Disziplin}

Die klinische Ethik ist eine relativ neue Disziplin in unseren westlichen Gesundheitssystemen. Es handelt sich bei dieser neuen Disziplin um eine angewandte, institutionalisierte Form von Ethik. Angewandt bedeutet, dass die ethischen Bemühungen sich um Vorschläge und Lösungen bemühen und nicht bei einer reinen Reflexion stehenbleiben (wollen). Und institutionalisiert bedeutet, dass die klinische Ethik als Disziplin - etwas salopp gesagt - in Organigrammen sichtbar wird. Krankenhäuser, Spitäler, Altersheime und andere Organisationen des Gesundheitswesens weisen der Ethik einen strukturierten, expliziten Platz in ihrer jeweiligen Institution zu. Diese Strukturierung der Ethik kann z.B. in Form einer hausinternen klinischen Ethikkommission erfolgen.* Solche klinischen Ethikkommissionen sind eine häufig anzutreffende Form der institutionalisierten Ethik in Gesundheitswesen der westlichen Welt. In der Regel setzen sich solche Ethikkommissionen interdisziplinär zusammen: Ärztinnen, Ärzte, Therapeutinnen, Pflegende, Seelsorger, aber auch Kaderpersonen aus der Verwaltung können vertreten sein. Manchen Kommissionen ist es wichtig, eine Juristin an Bord zu haben. Ich kenne aber auch Kommissionen, die bewusst auf die juristische Komponente verzichten und sich diese Expertise nur in Einzelfällen beratend hinzuziehen. Im westlichen Europa - schätzungsweise vor allem seit den 1980er Jahren entstanden - scheint eine typische Komponente von Ethikkommissionen deren Initiierung durch engagierte Mitarbeiter zu sein. Die meisten Kommissionen, die ich kenne, und die
Discussions de cas éthiques et "conseils" une introduction critique

En tant que discipline, l'éthique clinique s'entend communément comme un système visant à soutenir le personnel de la santé lors d'actes et de décisions difficiles sur le plan moral, par exemple sous la forme de discussions de cas éthiques. Mais ni la perception de ce que chacun ressent comme "éthiquement complexe» ni l'organisation et la tenue de discussions de cas éthiques ne sont triviales. Au contraire, les obstacles sont souvent si importants qu'il est plus facile de laisser l'éthique de côté que de l'institutionnaliser de manière judicieuse. Or les professionnels de la santé ne peuvent porter à eux seuls ces problèmes d'implémentation. Il est grand temps que les commissions éthiques cliniques et que les éthiciens se penchent sur leur propre activité et sur leur réflexion conceptuelle.

seit jener Zeit bestehen, sind «bottom-up» entstanden, aus dem ernsthaften und wohlgemeinten Engagement heraus, der eigenen Institution oder Abteilung ein geschärftes ethisches Profil im Kontext der zunehmend wahrgenommenen Ohnmacht von Spitzenmedizin verleihen zu wollen. Und viele dieser Kommissionen sind dann irgendwann «top-down» von der Geschäftsleitung oder Klinikleitung im Organigramm verankert worden. Ich kenne aber auch einige neuere Kommissionen, die direkt von der Geschäftsleitung "top-down" initiiert wurden. Und ich kenne einige Kommissionen, die ausgestorben sind. Sei es, weil sie 


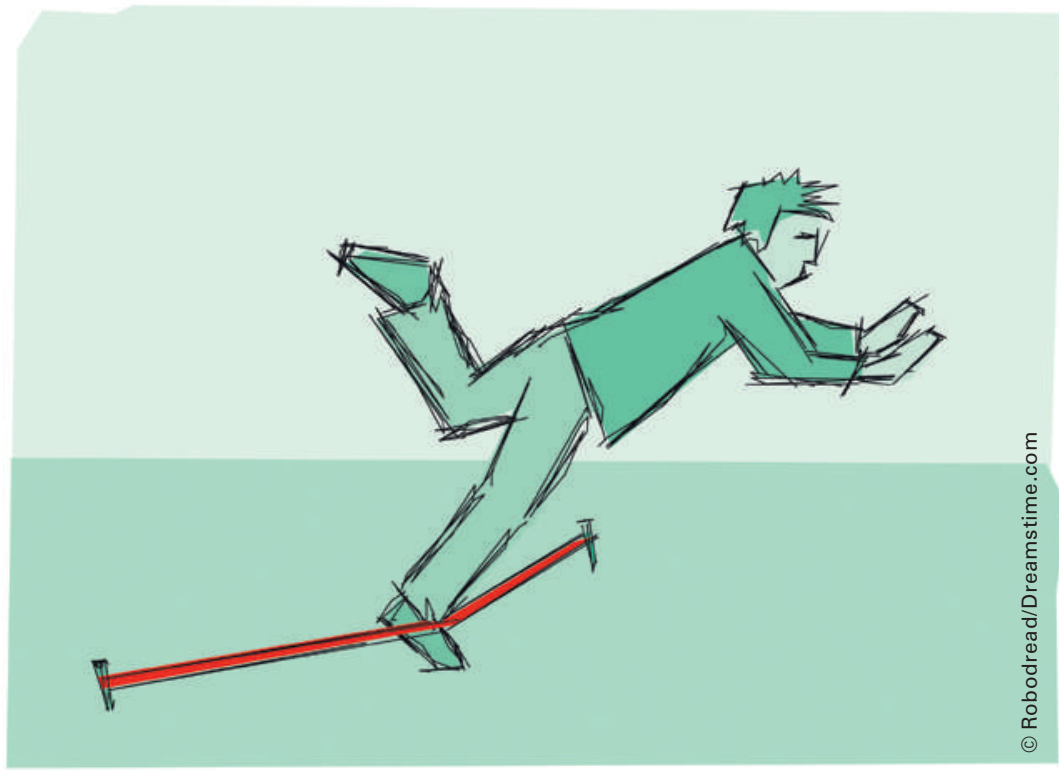

Ein möglicher Fallstrick bei Fallbesprechungen: die Erwartungshaltung, dass die Ethikkommission über die Lösung des problematischen Falles entscheidet.

ihre Aufgaben erfüllt sahen; weil die meisten, der von ihnen behandelten Themen plötzlich von Juristen aufgegriffen wurden; oder, und das scheint mir der häufigste Grund des Aussterbens, weil es ihnen nicht gelungen war, ihren neu geschaffenen Platz im Organigramm auch mit Leben auszufüllen. Und dabei wären wir beim Thema: Was machen klinische Ethikkommissionen eigentlich den ganzen Tag?

\section{Die Arbeit einer klinischen Ethikkommis- sion - Schwerpunkt Fallbesprechungen}

In der Regel treffen sich die Mitglieder von klinischen Ethikkommissionen nicht jeden Tag, sondern haben einen festen Rhythmus an Sitzungsdaten, sechsmal im Jahr, oder achtmal im Jahr. Es geht darum, ethische Empfehlungen und Richtlinien für die eigene Institution zu entwickeln, oder z.B. Weiterbildungsprogramme in Ethik zu initiieren und zu implementieren. Ein weiteres wichtiges gar genuines - Charakteristikum von Ethikkommissionen, bzw. der klinischen Ethik als Disziplin, ist die Idee, problematische Patientenfälle aus ethischer Sicht besprechen zu wollen. Die Rede ist von ethischen

Serie zum Thema "Klinische Ethik neu gedacht" In dieser SÄZ-Serie geht es um die Anforderungen und Herausforderungen der klinischen Ethik als neuer Disziplin im Gesundheitswesen. Die Beiträge sind bewusst subjektiv, interpretierend, aus Sicht eines klinischen Ethikers geschrieben und wollen zur Diskussion anregen. Der im Sommer erscheinende zweite Teil der Serie wird sich mit der Thematik «Paradigmenwechsel in der klinischen Ethik» befassen.
Fallbesprechungen. Solche Fallbesprechungen können z.B. akut durchgeführt werden, wenn irgendwo ein Patientenfall Probleme mit sich bringt (Probleme, die als ethisch-relevant angesehen werden). Dann wird z.B. eine Kontaktperson aus der Ethikkommission angerufen, und diese geht vor Ort, auf die Station, oder in die Abteilung, und führt eine ethische Fallbesprechung mit dem Team durch. Diese letzten drei Sätze wären ein eigenes Buch wert. Die Durchführung von Fallbesprechungen ist nämlich alles andere als trivial. Und manchmal frage ich mich, ob sich die Implementierung von klinischer Ethik nicht ganz anders entwickelt hätte, wenn nicht immer dieser Anspruch von Ethikkommissionen da gewesen wäre, schwierige Patientenfälle in akuten Fallbesprechungen direkt mitbehandeln zu wollen.

Eine erste Herausforderung ist die Wahrnehmung des Falles als ethisch-problematisch bzw. ethischrelevant. Pflegende oder Ärzte müssen also eine Art gedankliche Kategorie haben, wann sie etwas als ethisch-problematisch einordnen, bzw. wann sie es als relevant erachten, die Ethikkommission einschliessen zu wollen. Verschiedene Menschen haben allerdings verschiedene gedankliche Kategorien dazu, was ihnen als ethisch-problematisch erscheint, und was nicht. Ausserdem müssen die Gesundheitsfachpersonen gut verstehen, wann sie vielleicht nur ein mulmiges Bauchgefühl haben. Ganz oft hat so ein Bauchgefühl noch nichts mit einer ethischen Problemlage zu tun, sondern eher mit einem Unbehagen, Unverständnis oder mit einem interdisziplinären Kommunikationsproblem.

\section{Fallstricke bei Fallbesprechungen}

Manchmal ist das Bauchgefühl aber durchaus eine Intuition, die auf ein moralisches Problem hinweisen

\section{Was machen klinische Ethikkommissionen eigentlich den ganzen Tag?}

kann. Dann müssen diese Gesundheitsfachpersonen die Ethikkommission einschalten wollen. Das ist oft wie ein Eingeständnis ins eigene Versagen, schliesslich kommt man mit dem Patientenfall nicht zurecht. Und wenn es dennoch zum Einschalten der Ethikstruktur kommt, dann muss z.B. die Ethikkommission so strukturiert sein, dass sie - ehrlich gesagt im 24-Stunden-Rhythmus erreichbar sein muss. Medizinische Probleme richten sich kaum nach der Tageszeit. Dann muss eine Person (oder ein Team) aus der Ethikkommission möglichst unverzüglich in der Lage sein, sich ziemlich unmittelbar in die Abtei- 
lung zu begeben, um entweder das Behandlungsteam ethisch zu beraten, es anderweitig ethisch zu unterstützen bzw. um eine Fallbesprechung durchzuführen.

Wenn tatsächlich eine Fallbesprechung durchgeführt wird, dann müsste wieder vollkommen klar sein, dass alle Beteiligten wirklich mitmachen wollen, es müsste geklärt werden, welches Ergebnis mit der Durchführung der Fallbesprechung genau angestrebt wird, und z.B. auch, ob der Patient selbst (oder ein Angehöriger) teilnehmen will oder kann (beides wird in Europa eher selten gemacht). Es müsste auch klar sein, welche Rolle die Person aus der Ethikkommission genau wahrnimmt. Ist sie rein moderierend, oder wird von ihr erwartet, dass sie auch ethisch berät? Und wenn diese Person z.B. ein Arzt ist, dann müsste geklärt sein, dass dieser Arzt jetzt nicht als Arzt auftritt, sondern als Moderator der Ethikkommission. Diese Moderation der Fallbesprechung kann sich dann entweder nur auf den Besprechungsprozess konzentrieren, oder kann versuchen, ergebnisorientiert zu arbeiten. Auch das müsste geklärt sein. Manchmal wird von dem Ethikmoderator auch erwartet, dass er gar über die Lösung des problematischen Falles entscheidet. Ich finde, das ist eine völlig falsche Erwartung, und ich hoffe, dass Ethikmoderatoren oder Ethikkommissionen sich nicht in die Rolle drängen lassen, die Entscheidungsverantwortung für andere Gesundheitsfachpersonen zu übernehmen. Fazit: Die Prozesswege zur Durchführung von ethischen Fallbesprechungen sind alles andere als einfach. Ja, sie sind oft gar so kompliziert, dass ein Aussterben dieses Service-Angebotes der Ethikkommission wie vorprogrammiert zu sein scheint.

Meiner Meinung nach erfolgt die Implementierung von klinischen Ethikstrukturen viel besser über ein Weiterbildungsangebot für alle Mitarbeiter, anstatt sich direkt mit der Komplexität der Durchführung ethischer Fallbesprechungen zu verausgaben.

\section{Ethikkommissionen dürfen sich nicht in die Rolle drängen lassen, Entscheidungs- verantwortung für andere zu übernehmen.}

Greifen wir einen weiteren Punkt heraus, den ich im Kontext ethischer Fallbesprechungen auch immer wieder als problematisch erlebe: Wenn sich die Ethikkommissionen aus Mitgliedern zusammensetzen, die ihren klinischen Alltag als Ärztinnen, Pflegende und Therapeuten bestreiten, dann ist es diesen Personen oft allein aus zeitlichen Gründen gänzlich unmöglich, in akuten Situationen ihren Arzthut, oder
Pflegehut, schnell auszuziehen und sich den Hut der Ethikerin oder des Ethikers anzuziehen, um einen akuten Fall besprechen oder moderieren zu wollen. Deshalb sind einige Kommissionen tatsächlich auch wieder davon abgekommen, akute Fallbesprechungen durchzuführen. Sie behandeln die problematischen Fälle dann retrospektiv in ihren Kommissionssitzungen, und geben den Teams später Rückmeldungen oder Tipps, um besser mit zukünftigen, ähnlichen Fällen umgehen zu können.

\section{Die Implementierung von Ethikstrukturen erfolgt besser über Weiterbildungsangebote für Mitarbeiter statt über Fallbesprechungen.}

Eine andere Möglichkeit, akut auf Ethikfälle reagieren zu können, ist die Schaffung von ausgewiesenen Stellenprozenten für Ethikerinnen und Ethiker. Gerade in Universitätskrankenhäusern ist es zunehmend möglich, Einzelpersonen anzutreffen, die sich - entweder in einem Teilzeitpensum, oder Vollzeit angestellt - ganz der klinischen Ethik widmen. In der Regel sind diese ausgewiesenen klinischen Ethikerinnen und Ethiker an die Struktur der Ethikkommission angeschlossen, als deren Geschäftsführer, oder als Ethikreferent. Ich selbst arbeite als solch ein klinischer Ethiker, allerdings ohne Ethikkommission. Die Ethikkommission wurde in unserem Fall durch ein sogenanntes Netzwerk an Ethik-Ansprechpersonen ersetzt, insbesondere Pflegende und Ärztinnen, die selbst über Ausbildungen in Ethik verfügen, und die selbst vor Ort, auf Station, in den Kliniken, eigene Dienstleistungen in klinischer Ethik anbieten. Meine Rolle ist es dann, diese Personen zu koordinieren und professionell zu unterstützen.

\section{Von der Beratung ...}

Weiter oben habe ich schon mehrmals die beiden Verben beraten und unterstützen benutzt. Diese begriffliche Unterscheidung von dem, was eine Ethikkommission, bzw. eine Ethikerin, bieten kann, bedarf einer genaueren Betrachtung. Zunächst zum Beraten: Ethikberatung war lange Zeit ein gängiger Begriff für das, was klinische Ethik bieten kann. Ethikberatung ist eine deutsche Übersetzung des im Anglo-Amerikanischen immer noch sehr oft verwendeten Begriffes der "Ethics Consultancy». Ich glaube beobachten zu können, dass wir von dieser Vorstellung der Beratung immer mehr wegkommen. Im englischsprachigen Ethikumfeld begegnet man neuerdings immer mehr der Begrifflichkeit «Clinical Ethics Support» Services. Das könnte man im Deutschen als 
klinisch-ethische Unterstützung übersetzen. Dabei wären wir auch schon beim zweiten Verb, unterstützen, angelangt.

\section{... zur Unterstützung}

Als die Schweizerische Akademie für medizinische Wissenschaften, die SAMW, im Jahr 2009 eine Arbeitsgruppe eingesetzt hat, um für die Schweiz Richtlinien in der Implementierung und Durchführung von klinischer Ethik zu entwickeln, da begann die Arbeitsgruppe diese Arbeit unter dem gedanklichen Arbeitstitel durchzuführen, dass man Richtlinien zur Ethikberatung entwickeln wolle.* Nach drei Jahren Arbeitsgruppenarbeit, nach vielen Diskussionen, Überlegungen und Anhörungen von Experten, ist man letztendlich dazu übergegangen, lediglich Empfehlungen aussprechen zu wollen, und zwar unter dem Titel: Ethische Unterstützung in der Medizin. Man hat den Begriff der Unterstützung dem Titel der Beratung vorgezogen. $\mathrm{Zu}$ Recht, wie ich finde.

Warum jetzt dieser Fokus auf Begrifflichkeiten? Man könnte doch meinen, Begriffe wären nebensächlich, es ginge doch eigentlich um die Sache an sich. Ich denke, das stimmt nicht. Ich selbst glaube vielmehr, dass Begriffe und Konzepte gerade in einer neuen Disziplin sehr wichtig sind, und dass man sich noch viel mehr Gedanken machen müsste über präzisierte Begriffe und Konzepte. Die Unterscheidung zwischen Beratung und Unterstützung ist aus meiner Sicht nämlich nicht nur rein begrifflich zu verstehen, sondern sie transportiert auch einen normativ-konzeptuellen Anspruch des als Ethiker Auftretenden. Wenn ich mich als Berater versteheAchtung, ich übertreibe jetzt etwas -, dann verfüge ich in meiner eigenen Wahrnehmung offensichtlich über ein Wissen, das z.B. der in der täglichen praktischen Arbeit stehende Arzt nicht hat. Er wendet sich an mich, weil ihm die Kompetenz fehlt, in dieser ethisch-relevanten Situation alleine gut entscheiden zu können. Ich finde, das klingt ziemlich überheb- lich. Ich glaube sogar, dass die in der Praxis Tätigen ein viel grösseres Wissen über ihre Strukturen und Patienten haben, als es ein externes Ethikmitglied je erwerben kann. Aber weil sie so sehr in ihrer praktischen Arbeit am Krankenbett oder in ihrer Klinik involviert sind, sehen sie manchmal den Wald vor lauten Bäumen nicht. Dann braucht es die klinische Ethik, um hier unterstützend den Blick frei zu machen Letztendlich muss aber der Arzt, oder je nach Situation auch die Pflegende, wieder aus ihrer eigenen Berufsrolle heraus in der schwierigen Situation entscheiden können. Die klinische Ethik soll genau diese Reflexion unterstützen, nicht aber mit dem Anspruch kommen, dass sie die einzige ethische Kompetenz gepachtet hätte und diese in der Beratung ans Bett bringt bzw. vor Ort zur Verfügung stellt. Die klinische Ethik kann kein medizinischer Konsiliardienst sein, dafür entspricht sie zu wenig einer medizinischen Disziplin. Vielleicht karikiere ich hier etwas zu stark. Deshalb möchte ich als weiteres Pro-Argument zu Händen der Begrifflichkeit der Unterstützung weiter anfügen, dass ethische Unterstützung ein viel breiterer Begriff als Beratung ist. Zur Unterstützung kann man eher

\section{Die klinische Ethik soll die Reflexion} unterstützen und nicht beanspruchen, die einzige ethische Kompetenz zu haben.

auch die ganzen anderen Tätigkeiten rechnen, die in der klinischen Ethik betrieben werden, z.B. Lehre, Weiterbildungen, Richtlinienentwicklung usw. Der Begriff der Beratung ist mir zu sehr auf den klinischen Einzelfall ausgerichtet, die Begrifflichkeit der ethischen Unterstützung scheint mir offener, und das muss ich zugeben - sie scheint mir auch etwas bescheidener zu sein. Diese Unklarheit in den Begriffen symbolisiert aber durchaus auch eine Unklarheit, wie man klinische Ethik heutzutage überhaupt gut betreiben soll.

Dazu aber mehr im zweiten Teil dieser Artikelserie: «Paradigmenwechsel in der klinischen Ethik». 